\title{
Efeitos da nutrição in ovo no desempenho de frangos de corte: uma revisão
}

\author{
Effects of in ovo nutrition on broiler performance: a review \\ Efectos de la nutrición in ovo en el rendimiento de los pollos de engorde: una revisón
}

Recebido: 24/01/2021 | Revisado: 26/01/2021 | Aceito: 10/02/2021 | Publicado: 20/02/2021

\author{
Hemille Antunes Ferreira Miranda \\ ORCID: https://orcid.org/0000-0003-2218-1474 \\ Universidade Federal de Minas Gerais, Brasil \\ E-mail: hemilleantunes@gmail.com \\ Idael Matheus Góes Lopes \\ ORCID: https://orcid.org/0000-0002-1345-1084 \\ Universidade Federal de Minas Gerais, Brasil \\ E-mail: idael.matheus@gmail.com \\ Marcelo Dourado de Lima \\ ORCID: https://orcid.org/0000-0002-0454-7121 \\ Universidade Federal de Minas Gerais, Brasil \\ E-mail: mlima.2326@gmail.com \\ Fabiana Ferreira \\ ORCID: https://orcid.org/0000-0001-5827-0968 \\ Universidade Federal de Minas Gerais, Brasil \\ E-mail: ferreira_fabiana@outlook.com \\ Ellen Batista Pereira \\ ORCID: https://orcid.org/0000-0002-8270-2094 \\ Universidade Federal de Minas Gerais, Brasil \\ E-mail: elenbatista53@gmail.com \\ Luana Ferreira da Silva \\ ORCID: https://orcid.org/0000-0002-3324-3258 \\ Universidade Federal de Minas Gerais, Brasil \\ E-mail: luanaferreira3@outlook.com \\ Lorena Fernandes Costa \\ ORCID: https://orcid.org/0000-0003-1767-0054 \\ Universidade Federal de Minas Gerais, Brasil \\ E-mail: lorenafernandesc@hotmail.com
}

\begin{abstract}
Resumo
A eficiência na capacidade produtiva obtida por meio do melhoramento genético em frangos de corte, promoveu a necessidade de maior aporte nutricional embrionário. Para tal, a inoculação de soluções in ovo, contendo nutrientes e substâncias exógenas tornou-se uma importante técnica para aumentar as reservas energéticas e, consequentemente, obter melhor desempenho das aves pós eclosão. Sendo assim, objetivou-se com o presente trabalho de revisão, expor as principais metodologias e nutrientes utilizados na nutrição in ovo, mostrando os efeitos da técnica na produção de frangos de corte. Os carboidratos são utilizados como uma das principais fontes de energia para o embrião póseclosão, pois garantem o aumento na disponibilidade de glicose. Já as proteínas e aminoácidos são fundamentais para a eficiência da deposição proteica pós-eclosão. Os probióticos e prebióticos conferem melhor desenvolvimento e integridade do trato gastrointestinal, proporcionando melhor saúde intestinal aos animais. Por fim, o uso de vitaminas e minerais contribuem para a melhoria da imunidade dos animais, melhora no desenvolvimento esquelético durante período de incubação e pós eclosão, além de diversas outras funções. Visto isso, a utilização da nutrição in ovo pode garantir melhorias nos resultados zootécnicos de pintinhos no período pós-eclosão, pois contribui para melhor desempenho dos animais até o abate.
\end{abstract}

Palavras chave: Alimentação in ovo; Desenvolvimento embrionário; Eclosão; Nutrição embrionária; Desempenho.

\begin{abstract}
The productive efficiency obtained through broiler chicken' breeding promoted a greater need for embryonic nutritional supply. For this, the in ovo inoculation of solutions containing nutrients and exogenous substances has become an important technique to increase energy reserves and, consequently, obtain better performance of birds after hatching. Thus, our objective was to review both the methodologies and nutrients used at the in ovo nutrition. Furthermore, we focused on the effects of this technique on the broilers' production. Carbohydrates are used as one of the primary sources of energy for the post-hatch embryo, as they guarantee an increase in glucose availability. Proteins and amino acids are essential for the efficiency of protein deposition after hatching. Probiotics and prebiotics provide better gastrointestinal' development and integrity, and consequently, guarantee animals' intestinal health.
\end{abstract}


Finally, the use of vitamins and minerals contributes to animals' immunity, skeletal development during the incubation and post-hatch periods, between several other functions. Given this, in ovo nutrition techniques might promote zootechnical efficiency of broiler chicken in the post-hatch period, contributing to better performance of the animals until slaughter.

Keywords: Feed in ovo; Embryo development; Hatchability; Embryo nutrition; Performance.

\section{Resumen}

La eficiencia productiva obtenida a través del mejoramiento genético en pollos de engorde, promovió la necesidad de un mayor aporte nutricional embrionario. Para ello, la inoculación in ovo de soluciones que contienen nutrientes y sustancias exógenas se há convertido en una importante técnica para aumentar las reservas energéticas y, en consecuencia, obtener un mejor rendimiento de las aves tras la eclosión. Así, nuestro objetivo fue revisar tanto las metodologías como los nutrientes utilizados en la nutrición in ovo. Además, nos centramos en los efectos de esta técnica en la producción de los pollos de engorde. Los carbohidratos se utilizan como una de las principales fuentes de energía para el embrión post-nacimiento, ya que garantizan un aumento de la disponibilidad de glucosa. Las proteínas y los aminoácidos son esenciales para la eficacia de la deposición de proteínas tras la eclosión. Los probióticos y prebióticos proporcionan un mejor desarrollo e integridad gastrointestinal y, en consecuencia, garantizan la salud intestinal de los animales. Por último, el uso de vitaminas y minerales contribuye a la inmunidad de los animales, al desarrollo del esqueleto durante los periodos de incubación y post-nacimiento, entre otras muchas funciones. Por lo tanto, las técnicas de nutrición in ovo podrían promover la eficiencia zootécnica del pollo de engorde en el período posterior a la eclosión, contribuyendo a un mejor rendimiento de los animales hasta el sacrificio.

Palabras claves: Alimentación in ovo; Desarrollo del embrión; Incubabilidad; Nutrición del embrión; Rendimiento.

\section{Introdução}

A produção de carne de frango tornou-se o segmento de proteína animal que mais cresce mundialmente nos últimos anos, com aumento exponencial em seu consumo (Garcia; Gomes, 2019). O frango se tornou a carne mais consumida em virtude do alto valor nutricional, preço de mercado reduzido e maior quantidade de proteína produzida em curto espaço de tempo, quando comparado a outras espécies (De Meyer, et al., 2019).

Entretanto, quando se pensa no aumento da escala de produção é de extrema importância fornecer condições adequadas para que as aves produzam com máxima eficiência, e, para que isso ocorra, avanços tecnológicos, melhoramento genético, utilização de programas nutricionais e o bem-estar das aves devem ser levados em consideração, visto que tais fatores contribuem para alavancar a cadeia produtiva de frangos de corte (Rosalen et al., 2020).

Estudos na área de nutrição, tornam-se importantes para o bom desempenho das aves. Quando bem utilizadas, as estratégias nutricionais atuam positivamente na manutenção da saúde dos animais e como consequência promovem o bom desempenho dos mesmos (Givisiez, et al., 2020).

Dentre as diversas estratégias nutricionais utilizadas no mercado de produção avícola, a técnica da nutrição in ovo se apresenta como uma boa opção, pois consiste na inoculação de soluções, contendo nutrientes e substâncias de origem exógena que visam estabelecer fenótipos ao longo da vida, incluindo desempenho superior, imunidade e microbioma intestinal saudável nas aves pós-eclosão (Slawinska, et al., 2016; Siwek, et al., 2018).

Os benefícios na utilização da nutrição in ovo vem sendo comumente relatados por alguns autores na produção de frangos de corte (Alsultan, et al., 2020; Dal'Alba, et al., 2020), promovendo aumento no ganho de peso (Slawinska, et al., 2019), melhora no sistema imunológico (El-Deep, et al., 2020), melhorias intestinais nas aves após eclosão (Beck, et al., 2019; Skjøt-Rasmussen, et al., 2019), e como consequência melhor desempenho produtivo apresentado pelas mesmas (El-Moneim, et al., 2019).

Sendo assim, manter o foco em pesquisas que visem elucidar ainda mais os benefícios dessa técnica é de extrema importância, pois ajuda a impulsionar o âmbito da produção da avicultura de corte. Visto isso, a presente revisão tem como objetivo expor as principais metodologias e os principais nutrientes utilizados na nutrição in ovo bem como seus efeitos positivos na produção de frangos de corte. 


\section{Metodologia}

Foi utilizada a abordagem exploratória, com pressupostos da pesquisa bibliográfica e documental, segundo a metodologia proposta por Pereira, et al., (2018), tendo como produto uma revisão de literatura, compilando informações científicas relacionadas à temática das principais metodologias utilizadas na nutrição in ovo bem como os benefícios dessas no desempenho de frangos de corte. Fez-se seleção de artigos utilizando buscas bibliográficas no Portal da Capes, em bases a seguir: Scielo, Google Acadêmico, Science Direct e PubMed. A busca orientou-se com o emprego das palavras-chaves, alimentação in ovo, desenvolvimento embrionário, eclosão e nutrição embrionária. Posteriormente, realizou-se a seleção de teses, monografias e artigos, através de leitura criteriosa na redação dos textos. O período utilizado para escolha das pesquisas (2015 - 2020), ainda que tenham sido incluídos trabalhos abaixo da data estipulada, visto que a relevância deles foi significativa para escrita do tema proposto.

\section{Revisão de Literatura}

\subsection{Estrutura do ovo e desenvolvimento embrionário}

O período de incubação é considerado muito importante na produção de frangos de corte, pois corresponde a um terço do tempo de produção desses animais e problemas ocorridos nessa fase geram perdas irreparáveis. Aspectos físicos e fisiológicos precisam ser observados para o desenvolvimento embrionário ocorrer de forma adequada, finalizando com sucesso ao $21^{\circ}$ dia de incubação (Reis, 2018).

O crescimento e desenvolvimento do embrião é influenciado por manejo de incubação, sendo necessárias condições ótimas de temperatura, umidade, oxigenação e viragem dos ovos. O ovo fértil é composto macroscopicamente por casca, gema e albúmen, os quais fornecem os nutrientes necessários para sobrevivência e desenvolvimento do embrião até o momento da eclosão. Os ovos precisam passar por processo de classificação para ser garantida a maior qualidade dos pintinhos ao nascer (Alves, et al., 2020).

Nas aves, o desenvolvimento do embrião ocorre dentro do ovo, fora do corpo da ave, por esse motivo a nutrição do embrião depende exclusivamente dos componentes presentes no ovo. A qualidade da matriz e, consequentemente, do ovo fértil é o fator de maior importância na embriogênese, o processo no qual ocorre diferenciação celular, crescimento e maturação do embrião. Assim, ovos provenientes de matrizes saudáveis e bem nutridas fornecerão condições mais adequadas para o desenvolvimento embrionário_(Gonçalves, et al., 2013).

O anexo embrionário cuja principal função é armazenar reservas nutritivas para o embrião é o saco vitelínico, cuja composição nutricional corresponde a aproximadamente 32\% de lipídeos, $17 \%$ de proteínas, $1 \%$ de carboidratos e $50 \%$ de água. Os vasos sanguíneos apresentam crescimento nesse anexo e a formação de células do sangue e proliferação de célulastronco é iniciada. Depois da primeira semana de desenvolvimento embrionário o epitélio de absorção e a densa rede de capilares, incluirá completamente a gema (Kadam, et al., 2013).

O embrião é envolvido por membrana, denominada âmnio e a camada interna secreta o fluido denominado amniótico, conferindo proteção contra choques térmicos e mecânicos. Outra estrutura dos anexos embrionários importante é o córion, o qual envolve todas as estruturas embrionárias e serve como barreira protetora (Amaral, 2019).

O alantoide funciona como reservatório de resíduos metabólicos. Com o passar dos dias, esse funde-se com o córion, tornando-se a membrana cório-alantóide. Nos últimos dias de incubação, o embrião ocupa quase totalmente o ovo, o saco vitelínico é absorvido via cavidade abdominal e o embrião consome substâncias nutritivas importantes para o desenvolvimento. Posteriormente acontece o rompimento do âmnio e o embrião começa a respirar através da câmara de ar, sendo possível a bicagem e rompimento da casca para a eclosão (Amaral, 2019). 
O desenvolvimento do esqueleto embrionário é diretamente influenciado por dieta e idade das matrizes, pois estas depositam cálcio, fosforo, minerais e vitaminas no ovo. As linhagens modernas possuem maior exigência metabólica e muitas vezes é necessária suplementação exógena para suprir falhas nutricionais e garantir maior eficiência na fase de pós eclosão, pois a deficiência de nutrientes implica em consequências negativas no desenvolvimento das aves (Dal' Alba, 2018).

Em matrizes com idade avançada há aumento do peso e tamanho dos ovos, o que por sua vez reduz a perda de humidade e as trocas gasosas, diferentemente de matrizes jovens, as quais possuem ovos de casca mais espessa e o albúmen mais denso. Portanto, a idade da matriz pode influenciar na eclodibilidade. Embriões desenvolvidos em ovos maiores são menos tolerantes ao excessivo calor metabólico final da incubação (Rocha, 2011).

A fonte de cálcio disponível vem da casca do ovo, enquanto fósforo, minerais e vitaminas são oriundos da gema. A transferência materna de minerais vem sendo estudada, e em muitos casos, essa se torna insuficiente para o desenvolvimento ósseo embrionário (Torres, 2018).

\subsection{Técnica da nutrição in ovo}

Aproximadamente 40 anos atrás, a produção de frangos de corte passava por uma das mais importantes tecnificações. Em estudos feitos por Sharma e Burmester (1982), foram introduzidos os conceitos de vacinação in ovo, que visavam principalmente a imunização contra doenças, como é o exemplo da doença de Marek, que quando realizada a vacinação no período de incubação, a imunização seria de fato mais efetiva. Anteriormente, a vacina era mais comumente administrada no período neonatal, por via subcutânea no pescoço (Sharma \& Witter, 1983).

Após a primeira validação da viabilidade na utilização da tecnologia de vacinação in ovo, a tecnificação da indústria de produção avícola tornou-se importante para os ganhos positivos do setor, sendo possível otimizar os processos de incubação e eclosão na produção de frangos de corte através da vacinação em escala (Tainika \& Sekeroğlu, 2020). Além do uso com foco na prevenção de doenças, a vacinação in ovo é vista como uma alternativa para potencializar o desenvolvimento de frangos de corte, pois através desta é possível fornecer nutrientes para o embrião, que por sua vez irão promover boas respostas de desempenho ao longo do período produtivos dos mesmos (Gao, et al., 2017).

Em função do desenvolvimento embrionário nas aves ser realizado de forma separada do organismo materno, há a necessidade de suplementação extra de nutrientes para o bom desenvolvimento do embrião (Winjen, et al., 2020). Logo, o fornecimento exógeno de nutrientes através da nutrição in ovo irá suprir possíveis deficiências e exigências que acarretam má formação dos embriões bem como na redução das taxas de eclodibilidade (Saeed, et al., 2019), pois permite aumentar as reservas energéticas, acelerar o desenvolvimento do trato gastrointestinal do neonato (Roto, et al., 2016), melhorando os processos de absorção e digestão (Hollemans, et al., 2018), refletindo em melhor desempenho das aves pós eclosão (Gonzalez \& Jackson, 2020; Jha et al., 2019).

Diferentes métodos de administração e dosagens se mostraram eficientes para o uso da nutrição in ovo no plantel, variando em tamanho do ovo, tipo de ave, momento e local de inoculação. Dentre vários estudos, o local mais eficaz para injeção é o âmnio, sendo possível realizar o processo de forma manual ou automatizada (Peebles, 2018). No $18^{\circ}$ dia de incubação o embrião começa a realizar a ingestão do fluido amniótico para suprir gastos energéticos decorrentes do desenvolvimento corporal, tornando este momento propício para fornecer nutrientes exógenos que posteriormente serão depositados no intestino do embrião (Silva, 2016).

Portanto, o uso de nutrientes como os carboidratos (Neves, et al., 2020), aminoácidos (Han, et al., 2020), vitaminas e minerais (Shokraneh, et al., 2020; Teymouri, et al., 2020), e aditivos, probióticos e prebióticos (Dankowiakowska, et al., 2019; Li, et al., 2020), suprem as demandas nutricionais do embrião, bem como proporcionam às aves condições favoráveis para expressarem o potencial produtivo no período pós-eclosão. 


\subsubsection{Principais nutrientes utilizados na nutrição in ovo}

\section{Carboidratos}

Ao final da incubação, os embriões absorvem uma reserva energética do saco vitelínico, porém, essa pode não ser suficiente para suprir as exigências de carboidratos necessárias pós eclosão, porque a reserva de carboidratos do saco vitelínico é baixa, menos de $1 \%$ do total, e desse apenas $0,3 \%$ é glicose livre (Damasceno, et al., 2017). Logo, a necessidade de suplementar fontes de carboidratos torna-se importante. Um exemplo é o glicerol, pois quando suplementado via injeção in ovo, contribui fornecendo energia para o embrião no período de incubação e para o pintinho pós-eclosão (Neves, et al, 2016).

Dal Pont, et al., (2019), citam que a suplementação in ovo de glicerol favorece o desenvolvimento do embrião no período de incubação, além de promover aumento no armazenamento de glicogênio hepático em pintinhos recém-nascidos. Entretanto, é indispensável levar em consideração o local de inoculação e principalmente as doses a serem injetadas, pois fatores como estes irão condizer se a técnica de suplementação de tal nutriente é de fato benéfica (Kadam, et al., 2013).

Leitão, et al. (2014), utilizando maltose e sacarose inoculados ao $16^{\circ}$ dia de incubação avaliaram eclodibilidade, maturidade gastrointestinal e desempenho até 14 dias de idade de pintinhos de corte. Nesse estudo não houve interferência no peso ao nascer e eclodibilidade, mas $0,6 \mathrm{ml}$ de maltose favoreceram maior altura de vilosidades no primeiro dia de vida, sem diferença de desempenho na fase inicial.

Pensando nisso a inoculação de carboidratos através da nutrição in ovo foi testada durante a incubação de ovos férteis (Neves, 2019; Berrocoso, et al., 2016; Nunes, et al., 2018; Bhanja, et al., 2014; Kanagaraju \& Rathnapraba, 2019), visando se avaliar os locais de inoculação, as dosagens, os benefícios dessa técnica para o embrião bem como o desempenho que proporciona na produção de frangos de corte (Tabela 1).

Tabela 1 - Pesquisas envolvendo a inoculação de carboidratos in ovo.

\begin{tabular}{|c|c|c|c|c|}
\hline Carboidratos & Dosagem & $\begin{array}{l}\text { Local e dia de } \\
\text { Aplicação } \\
\text { (incubação) }\end{array}$ & Resultados & Autor \\
\hline $\begin{array}{l}\text { Glicerol; Fator de } \\
\text { crescimento } \\
\text { semelhante à } \\
\text { insulina (IGF-I) }\end{array}$ & $\begin{array}{l}10 \mathrm{nmol} / \mathrm{mL} \text { de glicerol } \\
100 \mathrm{ng} / \mathrm{mL} \text { de IGF-I }\end{array}$ & Âmnio - $17^{\circ}$ dia & $\begin{array}{l}\text { Aumento no número de } \\
\text { ovos eclodidos e } \\
\text { melhora no desempenho } \\
\text { das aves pós eclosão }\end{array}$ & Neves, (2019) \\
\hline Rafinose & $\begin{array}{l}\text { Solução de Rafinose: } \\
1,5 ; 3,0 \text { e } 4,5 \mathrm{mg} \text { em } 0,2 \\
\text { mL de um diluente } \\
\text { aquoso }\end{array}$ & Saco de ar $-12^{\circ}$ dia & $\begin{array}{l}\text { Melhora na morfologia } \\
\text { da mucosa e na } \\
\text { imunidade do intestino } \\
\text { delgado de pintinhos de } \\
\text { corte }\end{array}$ & Berrecoso, et al., (2016) \\
\hline $\begin{array}{l}\text { Glicose e clara de } \\
\text { ovo líquida }\end{array}$ & $\begin{array}{c}0,5 \mathrm{ml} \text { de solução salina } \\
0,9 \%+200 \mathrm{mg} \text { de } \\
\text { glicose; } 0,5 \mathrm{ml} \mathrm{de} \\
\text { solução salina } 0,9 \%+ \\
0,25 \mathrm{ml} \text { de clara de ovo } \\
\text { líquida }\end{array}$ & $\begin{array}{l}\text { Líquido Âmniótico - } \\
17^{\circ} \text { dia }\end{array}$ & $\begin{array}{c}\text { Melhora no desempenho } \\
\text { de crescimento e peso } \\
\text { das aves }\end{array}$ & $\begin{array}{l}\text { Da Silva Nunes, et al., } \\
\qquad(2018)\end{array}$ \\
\hline $\begin{array}{l}\text { Glicose, Frutose e } \\
\text { Ribose }\end{array}$ & $\begin{array}{l}\text { Solução injetável de } \\
\text { Glicose, Frutose e } \\
\text { Ribose na dose de } \\
\text { 50mg/ovo }\end{array}$ & $\begin{array}{l}\text { Saco Vitelino / } \\
\text { Âmnio - } 14^{\circ} \text { dia }\end{array}$ & $\begin{array}{l}\text { Melhora no crescimento } \\
\text { e na imunidade celular } \\
\text { em frangos de corte }\end{array}$ & Bhanja, et al., (2014) \\
\hline
\end{tabular}




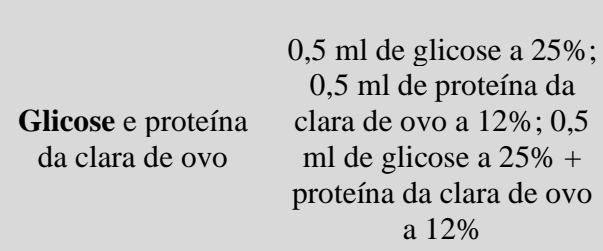

$0,5 \mathrm{ml}$ de glicose a $25 \%$; clara de ovo a $12 \% \cdot 0,5$ $\mathrm{ml}$ de glicose a $25 \%+$ a $12 \%$

Fonte: Autores (2021).

A suplementação isolada e a combinação de ambos promoveram melhora no desempenho produtivo e na histomorfologia intestinal de frangos de corte

A Tabela 1 traz a análise geral dos trabalhos mencionados, com base nas informações descritas pelos autores, apresentando os principais carboidratos utilizados, os benefícios apresentados, bem como a dosagem, local e dia de aplicação.

\section{Proteína e aminoácidos}

A formulação de dietas para monogástricos é feita com base no conceito de proteína ideal, sendo essa o balanço de aminoácidos em proporções exatas para atender às exigências de manutenção e crescimento dos animais (Van Milgen \& Dourmad, 2015). As utilizações de dietas equilibradas diminuem custos adicionais à formulação e a excreção de nitrogênio, pois o aumento deste pode acarretar problemas sanitários e reduzir o desempenho dos animais (Dorigam, 2016).

A maioria dos componentes do albúmen do ovo são inteiramente proteicos e as poedeiras possuem demanda maior de proteína e aminoácidos. A carência desses nutrientes ocasiona diminuição na quantidade de albúmen e no tamanho do ovo (Schmidt et al., 2011) e, consequentemente, interfere negativamente no acesso do embrião a esses nutrientes. As concentrações de aminoácidos presentes na gema não são limitantes para o crescimento do embrião, contudo, com a adoção de linhagens genéticas de animais com crescimento acelerado, consequência do melhoramento genético, a suplementação destes nutrientes se torna importante nas fases embrionária e pós eclosão dos frangos de corte (Maia, 2018).

A produção industrial possibilita acesso aos aminoácidos DL-metionina, L-lisina, L-treonina, L-triptofano e L-Valina e a formulação de dietas passou a ser realizada com base no conceito de proteína ideal. Com a disponibilidade desses aminoácidos industriais, a inclusão destes compostos tem sido prática rotineira nas fábricas de rações para aves (Costa et al., 2014), assim como estudos realizados sobre o efeito do uso dos aminoácidos na nutrição in ovo (Tabela 2). 
Tabela 2 - Pesquisas envolvendo a inoculação de aminoácidos in ovo.

\begin{tabular}{|c|c|c|c|c|}
\hline Aminoácidos & Dosagem & $\begin{array}{c}\text { Local e dia de } \\
\text { Aplicação (incubação) }\end{array}$ & Resultados & Autor \\
\hline L-arginina & $\begin{array}{l}\text { Inoculação de } 0,5 ; 1,0 ; \text { e } \\
1,5 \% \text { de L-arginina }\end{array}$ & Albúmen - $10^{\circ}$ dia & $\begin{array}{l}\text { Aumento de peso e } \\
\text { circunferência do } \\
\text { embrião, ganho de peso } \\
\text { corporal e redução na } \\
\text { conversão alimentar }\end{array}$ & Azhar, et al., (2016) \\
\hline L-lisina & $\begin{array}{c}\text { 10, 20,30, } 40 \text { e } 50 \mathrm{mg} \\
\text { de lisina em } 1 \mathrm{ml} \mathrm{de} \\
\text { água estéril }\end{array}$ & $\begin{array}{l}\text { Líquido amniótico - } \\
14^{\circ} \mathrm{dia}\end{array}$ & $\begin{array}{l}\text { Uso de } 20 \mathrm{mg} \text { de L- } \\
\text { lisina apresentou } \\
\text { melhores resultados } \\
\text { quanto a crescimento do } \\
\text { intestino delgado e } \\
\text { desenvolvimento do } \\
\text { músculo esquelético }\end{array}$ & Ebrahimi, et al., (2017) \\
\hline Treonina & $\begin{array}{l}0,05 \text { ou } 0,1 \mathrm{ml} \mathrm{de} \\
\text { solução salina (diluente) } \\
\text { com ou sem THR ( } 5 \mathrm{mg} \\
\qquad / \mathrm{ml})\end{array}$ & Câmara de ar $-11^{\circ}$ dia & $\begin{array}{l}\text { Melhora na morfologia } \\
\text { intestinal dos animais } \\
\text { recém eclodidos }\end{array}$ & $\begin{array}{l}\text { Kermanshahi, et al., } \\
\text { (2017) }\end{array}$ \\
\hline $\begin{array}{l}\text { Arginina }(\mathrm{Arg})+ \\
\text { Treonina }(\mathrm{Thr})\end{array}$ & $\begin{array}{l}\text { Solução injetável }(0,5 \\
\text { ml/ovo) contendo } 35 \mathrm{mg} \\
\text { Arg }+25 \mathrm{mg} \text { de } \mathrm{Thr} \\
\text { dissolvidas em solução } \\
\text { salina a } 0,5 \%\end{array}$ & Âmnio - $14^{\circ}$ dia & $\begin{array}{l}\text { Melhora na resposta } \\
\text { imune e aumento no } \\
\text { peso de órgãos linfoide } \\
\text { no período pós eclosão } \\
\text { em frangos de corte }\end{array}$ & Toghyani, et al., (2018) \\
\hline L-arginina & $\begin{array}{c}\text { Solução injetável } \\
\text { contendo } 0,5 \% \text { de L- } \\
\text { arginina }\end{array}$ & $\begin{array}{l}\text { Líquido amniótico }-14^{\circ} \\
\text { dia }\end{array}$ & $\begin{array}{l}\text { Aumento no consumo } \\
\text { de ração, redução na } \\
\text { taxa de conversão } \\
\text { alimentar e melhorias na } \\
\text { microflora cecal }\end{array}$ & Omidi, et al., (2019) \\
\hline
\end{tabular}

Fonte: Autores (2021).

A Tabela 2 traz a análise geral dos trabalhos mencionados, com base nas informações descritas pelos autores, apresentando os principais aminoácidos inoculados, os benefícios apresentados, bem como a dosagem, local e dia de aplicação.

A lisina é utilizada como referência, apesar de ser o segundo aminoácido limitante para aves, e possui metabolismo orientado principalmente para deposição proteica. Além disso, apresenta análise laboratorial relativamente simples, sendo a suplementação em dietas de aves economicamente viável (Martins et al., 2018).

Coskun, et al., (2018), inocularam ao $16^{\circ}$ dia de incubação lisina, metionina e a associação destes aminoácidos, com o objetivo de avaliar os efeitos sobre a eclodibilidade, desenvolvimento gastrointestinal e desempenho de crescimento do animal. 
Ao avaliarem os resultados, observou-se que o tratamento com lisina houve maior taxa de eclosão e o efeito foi positivo no comprimento do trato gastrointestinal.

Testes realizados por Yu, et al., (2017), objetivaram avaliar os efeitos da injeção in ovo de L-arginina sobre o metabolismo energético de frangos de corte pós-nascimento. Com este estudo, pode-se evidenciar que a suplementação com Larginina a $1,0 \%$ injetada no líquido amniótico no $17^{\circ}$ dia de incubação proporcionou aumento nas reservas energéticas do fígado e do músculo peitoral após a eclosão, deste modo regulando o metabolismo energético inicial em frangos de corte.

Em estudos feitos com injeção in ovo da treonina em frangos de corte desafiados com Salmonella, Moreira Filho, et al., (2018), consideraram a contagem bacteriana no conteúdo cecal, morfologia intestinal e ganho de peso do animal, e identificaram melhora no desempenho dos animais, maior peso à eclosão e redução da colonização por Salmonella enteritidis.

Em outro trabalho, Han, et al., (2020), testaram a inoculação in ovo de L-leucina sobre a termotolerância, crescimento e metabolismo de aminoácidos em frangos de corte sob estresse térmico. Ao término do trabalho os autores evidenciaram que a suplementação in ovo com L-leucina proporcionou maior termotolerância ao estresse por calor em frangos de corte além de contribuir para maior ganho de peso corporal diário.

A utilização de alguns aminoácidos nas dietas animais pode favorecer o bom funcionamento do trato gastrintestinal. Pensando nisso, Wang, et al., (2020), observaram os efeitos da inoculação in ovo de N-acetil-L-glutamato (NAG), no desenvolvimento intestinal inicial bem como o desempenho de frangos de corte. Ao término do trabalho, foi evidenciado que a inoculação de NAG (1,5 mg/ovo), acelerou o desenvolvimento intestinal, melhorando a função de absorção e imunidade do intestino, e, consequentemente, proporcionou melhor eficiência alimentar das aves nas duas primeiras semanas pós eclosão.

\section{Vitaminas e minerais}

A deficiência de nutrientes como os minerais pode ocasionar mal desenvolvimento embrionário esquelético e do sistema imune dos pintinhos. A deposição de cálcio, fósforo e minerais como cobre, zinco e manganês, juntamente com a vitamina D são responsáveis por auxiliar no desenvolvimento esquelético embrionário. A principal fonte de cálcio vem da própria casca do ovo, enquanto os outros minerais e vitaminas são derivados da gema do ovo (Torres \& Korver, 2018). Alguns estudos foram realizados com a utilização desses produtos na nutrição in ovo (Tabela 3). 
Tabela 3 - Pesquisas envolvendo a inoculação de Vitaminas e Minerais in ovo.

\begin{tabular}{|c|c|c|c|c|}
\hline Vitaminas e Minerais & Dosagem & $\begin{array}{c}\text { Local e dia de } \\
\text { Aplicação (incubação) }\end{array}$ & Resultados & Autor \\
\hline $\begin{array}{l}\text { Diluente comercial } \\
\text { contendo microminerais } \\
\text { (Zinco, Manganês e } \\
\text { Cobre) }\end{array}$ & $\begin{array}{l}\text { Diluente contendo } \\
0,544 ; 0,260 \text { e } 0,030 \\
\text { mg/mL de Zinco, } \\
\text { Manganês e Cobre. }\end{array}$ & Âmnio $-17^{\circ}$ dia & $\begin{array}{l}\text { Atuou positivamente na } \\
\text { mineralização óssea em } \\
\text { frangos de corte }\end{array}$ & Oliveira, et al., (2015) \\
\hline Zinco & $\begin{array}{c}\text { Injeção in ovo de } 50 \text { e } \\
100 \mu \mathrm{g} \text { Zinco/Ovo }\end{array}$ & Saco vitelino $-18^{\circ}$ dia & $\begin{array}{l}\text { Redução na taxa de } \\
\text { mortalidade } \\
\text { embrionária, aumento } \\
\text { na eclodibilidade e no } \\
\text { número de pintinhos } \\
\text { saudáveis }\end{array}$ & Sun, et al., (2018) \\
\hline $\begin{array}{c}\text { Zinco }(\mathrm{Zn}), \text { Cobre }(\mathrm{Cu}), \\
\text { Magnésio }(\mathrm{Mn})\end{array}$ & $\begin{array}{c}80 \mu \text { g.egg-1 de } \mathrm{Zn} \\
\text { inorgânico; } 6 \mu \text { g.egg.-1 } \\
\mathrm{Cu} \text { inorgânico, } 0,3 \\
\mu \mathrm{g} \text {.egg.-1 de } \mathrm{Mn} \\
\text { inorgânico }\end{array}$ & Âmnio - $18^{\circ}$ dia & $\begin{array}{l}\text { Aumento na taxa de } \\
\text { eclodibilidade, melhora } \\
\text { no crescimento do } \\
\text { coração, na } \\
\text { porcentagem do } \\
\text { duodeno e da largura do } \\
\text { eixo médio da tíbia }\end{array}$ & Sahr, et al., (2020) \\
\hline Vitamina $\mathrm{C}$ & $\begin{array}{c}3 \mathrm{mg} \text { de vitamina C } \\
\text { dissolvido em } 0,1 \mathrm{~mL} \\
\text { de solução salina } \\
\text { normal }\end{array}$ & Saco Vitelino $-11^{\circ}$ dia & $\begin{array}{l}\text { Melhora no desempenho } \\
\text { pós nascimento e } \\
\text { melhora no estado } \\
\text { imunológico em frangos } \\
\text { de corte }\end{array}$ & Zhu, et al., (2020) \\
\hline $\begin{array}{l}\text { Vitamina } \mathrm{E} \\
\text { de girassol) }\end{array}$ & $\begin{array}{l}\text { Inoculação in ovo de } 0,5 \\
\text { mL de óleo de girassol, } \\
\text { com vários níveis de } \\
\text { concentração de } \\
\text { Vitamina E }(0,0 ; 27,5 \\
38 ; 5,49,5 \text { e } 60,4 \mathrm{IU})\end{array}$ & $\begin{array}{l}\text { Cavidade Âmniótica - } \\
17^{\circ} \mathrm{dia}\end{array}$ & $\begin{array}{l}\text { Melhora no estado } \\
\text { oxidativo dos pintinhos } \\
\text { e consequentemente } \\
\text { melhorias nos resultados } \\
\text { de incubação e } \\
\text { desempenho }\end{array}$ & Araújo, et al., (2018) \\
\hline
\end{tabular}

Fonte: Autores (2021).

A Tabela 3 traz a análise geral dos trabalhos mencionados, com base nas informações descritas pelos autores, apresentando as principais vitaminas e minerais inoculados, os benefícios apresentados, bem como a dosagem, local e dia de aplicação.

A deposição de minerais in ovo é importante para o bom desenvolvimento embrionário, esquelético, muscular e imunológico. A suplementação de minerais e vitamina D3 mostrou-se benéfica, pode induzir maior consumo, e melhorar também as propriedades ósseas dos frangos na fase inicial (Yair, et al., 2015).

Joshua, et al. (2016), suplementando in ovo nanopartículas de zinco, cobre e selênio, aos 18 dias de incubação, observaram melhor desempenho pós nascimento, apresentando melhor eficiência alimentar e deposição muscular, não interferindo significativamente na eclodibilidade.

Em pesquisa realizada por Biria, et al., (2020), foi avaliado os efeitos sobre taxa de eclodibilidade e eficiência de desempenho em frangos de corte pós eclosão, através da suplementação in ovo de nanopartículas de zinco (50, 75 e 100 ppm). 
Ao término do trabalho, os autores evidenciaram que a suplementação do produto proporcionou aumento na taxa de eclodibilidade bem como maior consumo de ração na fase inicial.

Araújo (2017), suplementou diferentes níveis de Vitamina E in ovo, com o objetivo de melhorias nos resultados de incubação e desempenho inicial de frangos de corte e a utilização da vitamina E promoveu melhora na eclodibilidade, diminuiu o intervalo de nascimentos e melhorou a qualidade física dos animais.

Outro tipo de vitamina bastante importante a ser suplementada para aves é a vitamina $\mathrm{C}$, o ácido ascórbico, pois possui capacidade de modular o sistema de defesa antioxidante e imunológico (El-Senousey, et al., 2018), proporcionando melhores índices zootécnicos na produção de frangos de corte (Zhang, et al., 2019). Estudos realizados para testar a inoculação in ovo com vitamina $\mathrm{C}(3 \mathrm{mg} / \mathrm{ovo})$, no $15^{\circ}$ dia de incubação, sobre o desempenho de crescimento e função imunológica de frangos de corte, obtiveram como resultados melhora na taxa de eclodibilidade e aumento nos níveis de imunoglobulina (IgM), além de melhorar a taxa de crescimento dos animais (Zhu, et al., 2019).

\section{Prebióticos e Probióticos}

Os prebioticos e probióticos são utilizados na alimentação de frangos de corte e galinhas poedeiras visando melhorias à saúde intestinal dos animais (Wang, et al., 2017). Estes produtos são equilibradores da microbiota intestinal, desencadeiam crescimento da população microbiana benéfica e do sistema imunológico local, tendo consequências positivas sobre o ganho de peso do animal (Reis \& Vieites, 2019), além de serem uma alternativa frente ao uso de antibióticos (Agyare, et al., 2018).

Esses produtos são considerados micro-organismos vivos e quando administrados nas quantidades corretas, promovem controle da população de patógenos, melhoram saúde do hospedeiro (Adhikari \& Kim, 2017), e, consequentemente o desempenho animal, principalmente em animais monogástricos (Castañeda, et al., 2020; Lima, et al., 2020). Os principais com efeito positivo sobre o desempenho de frangos de corte são dos gêneros Lactobacillus, Enterococcus, Streptococcus, Bacillus e leveduras (Reis \& Vieites, 2019). Estudos foram realizados com alguns destes produtos, visando obter informações sobre os benefícios que os mesmos podem agregar na produção de frangos de corte (Tabela 4). 
Tabela 4 - Pesquisas envolvendo a inoculação de Prebióticos e Probióticos in ovo.

\begin{tabular}{|c|c|c|c|c|}
\hline Prebióticos, Probióticos & Dosagem & $\begin{array}{c}\text { Local e dia de } \\
\text { Aplicação (incubação) }\end{array}$ & Resultados & Autor \\
\hline Prebióticos e simbióticos & $\begin{array}{c}\text { Bi2tos 0,528 mg } \\
\text { (galacto- } \\
\text { oligossacarídeos) e } \\
\text { inulina 1,76 mg (frutano) } \\
\text { + Lactococcus lactis } \\
\text { subsp. lactis }\end{array}$ & Câmara de ar - $12^{\circ}$ dia & $\begin{array}{c}\text { Aumento no peso } \\
\text { corporal final }\end{array}$ & $\begin{array}{c}\text { Pruszynska-Oszmalek, et } \\
\text { al., (2015) }\end{array}$ \\
\hline
\end{tabular}

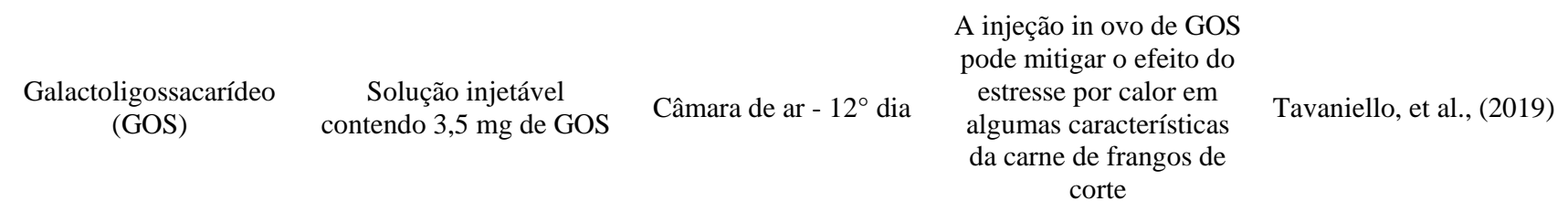

\begin{tabular}{|c|c|c|c|c|}
\hline $\begin{array}{c}\text { Prebiótico (trans- } \\
\text { galactoligossacarídeo, } \\
\text { Bi2tos) }\end{array}$ & $\begin{array}{c}\text { Solução injetável } \\
\text { contendo } 200 \mathrm{uL} \mathrm{de} \\
\text { solução salina }+3,5 \mathrm{mg} \\
\text { de trans- } \\
\text { galactoligossacarídeo } \\
\text { Bi2tos }\end{array}$ & Câmara de ar - $12^{\circ}$ dia & $\begin{array}{l}\text { Redução na gravidade } \\
\text { das lesões intestinais } \\
\text { causadas por infecção } \\
\text { induzida por Eimeria e } \\
\text { melhora no desempenho } \\
\text { de crescimento }\end{array}$ & Angwech, et al., (2019) \\
\hline
\end{tabular}

Fonte: Autores (2021).

A Tabela 4 traz a análise geral dos trabalhos mencionados, com base nas informações descritas pelos autores, apresentando os principais prebióticos e probióticos utilizados, os benefícios apresentados, bem como a dosagem, local e dia de aplicação.

Avaliando os efeitos da inoculação in ovo de Bacillus subtilis sobre o desempenho de crescimento e morfologia intestinal de frangos de corte, Oladokun, et al., (2020) observaram que a inoculação de tal produto não trouxe ganhos positivos ao desempenho das aves, entretanto, proporcionou melhora na morfologia intestinal dos animais. Em outro estudo foram testados prebióticos e simbióticos in ovo no $12^{\circ}$ dia de incubação de frangos de corte. Os resultados obtidos foram favoráveis, sendo observado aumento das vilosidades no jejuno e duodeno e aumento de células caliciformes no intestino delgado de pintinhos de um aos quatro dias de vida (Bogucka, et al., 2016).

O uso de substâncias prebióticas é importante para conferir melhorias na saúde do trato gastrointestinal e otimizar o desempenho dos animais (Neveling \& Dicks, 2020). O prebiótico DiNovo®, extrato da espécie Laminaria de alga marinha, injetado in ovo ao $12^{\circ}$ dia de incubação, aumentou significativamente o tamanho das vilosidades duodenais e profundidade das criptas, com influência maior sob os parâmetros histomorfológicos dos animais aos 21 dias pós eclosão (Sobolewska, et al., 2017). Em outra pesquisa, Dankowiakowska, et al., (2019), testaram a inoculação in ovo de prebióticos e simbióticos, no $12^{\circ}$ dia de incubação de embriões de frango de corte, visando observar os efeitos sobre os parâmetros de produtividade e qualidade da carne dos frangos abatidos aos 35 dias de idade. Ao término da pesquisa os autores observaram que a utilização in ovo dos produtos proporcionou efeito positivo sobre o peso corporal das aves e qualidade da carne.

Miśta, et al. (2017), observaram que o tratamento contendo simbióticos promoveu aumento sob a relação comprimento de vilosidades e profundidade de criptas na mucosa jejunal, o que por sua vez pode contribuir para aumento no peso corporal das aves, em consequência da melhor capacidade de absorção dos nutrientes. Martins (2018), constatou redução 
significativa da contaminação por Salmonella eidelberg no conteúdo cecal além de melhorar a integridade da mucosa entérica de frangos de corte, quando desafiados por Salmonella.

\section{Considerações Finais}

Diante dos estudos analisados no presente trabalho, percebeu-se que devido aos avanços no melhoramento genético em frangos de corte, os animais passaram a ter maior demanda por nutrientes durante o período embrionário e de produção. Logo, o fornecimento de nutrientes durante o período de incubação através da técnica da nutrição in ovo, proporcionou melhores resultados pós eclosão, além de influenciar positivamente no desempenho dos animais até o abate. Sendo assim, a nutrição in ovo apresenta-se como técnica agregadora de qualidade e desempenho do pintinho neonato, sendo importante principalmente como aporte de nutrientes no período de maior gasto energético, o momento de eclosão.

Entretanto, mesmo sendo técnica empregada em alguns incubatórios, maiores estudos são necessários para determinar quais nutrientes ou misturas de nutrientes inocular, para alcançar os benefícios almejados, pois os resultados apresentados ainda possuem divergências. Além disso, há a necessidade de realização de novos estudos, para avaliar a diversidade de opções existentes na execução da técnica, bem como entender melhor a funcionalidade e aplicabilidade desta.

\section{Referências}

Adhikari, P. A., \& Kim, W. K. (2017). Overview of Prebiotics and Probiotics: Focus on Performance, Gut Health and Immunity - A Review. Annals of Animal Science, 17(4), 949-966. 10.1515/aoas-2016-0092.

Agyare, C., Etsiapa Boamah, V., Ngofi Zumbi, C., \& Boateng Osei, F. (2019). Antibiotic Use in Poultry Production and Its Effects on Bacterial Resistance. Antimicrobial Resistance - A Global Threat. 10.5772/intechopen.79371.

Alsultan, O. M., Al-Khafaji, F. R. A., \& Gmash, H. N. (2020). Effects of combining in ovo injection by nutritive solutions and early post-hatch nutrition on productive performance of broiler. Plant Archives, 20(2), 1584-1591. http://www.plantarchives.org/SPL\%20ISSUE\%2020-2/261_1584-1591_.pdf.

Alves, L. K. S., Viana, G. P., Santos, T. S., Reis, B. Q. dos, Guimarães, E. B. B., Nascimento, R. A., Raineri, C., \& Araújo, C. S. S. (2020). In-ovo feeding: a review. Veterinária Notícias, 26(1), 18-18. http://www.seer.ufu.br/index.php/vetnot/article/view/51611/29670.

Amaral, V. T. (2019). Incubação de ovos férteis e o desenvolvimento embrionário. Trabalho de Conclusão de Curso (Bacharelado em Zootecnia) - Unidade Acadêmica de Garanhuns, Universidade Federal Rural de Pernambuco, Garanhuns. p. 33. https://repository.ufrpe.br/handle/123456789/1920.

Angwech, H., Tavaniello, S., Ongwech, A., Kaaya, A. N., \& Maiorano, G. (2019). Efficacy of In Ovo Delivered Prebiotics on Growth Performance, Meat Quality and Gut Health of Kuroiler Chickens in the Face of a Natural Coccidiosis Challenge. Animals, 9(11), 876. 10.3390/ani9110876.

Araújo, I. C. S. (2017). Suplementação in ovo de vitamina E e cantaxantina para embriões de frango de corte. Tese (Doutorado em Zootecnia) - Universidade Federal de Goiás, Goiânia. p. 71. https://repositorio.bc.ufg.br/tede/handle/tede/8003.

Araújo, I. C. S., Café, M. B., Noleto, R. A., Martins, J. M. S., Ulhoa, C. J., Guareshi, G. C., Reis, M. M., \& Leandro, N. S. M. (2018). Effect of vitamin E in ovo feeding to broiler embryos on hatchability, chick quality, oxidative state, and performance. Poultry Science, 98(9), 3652-3661. 10.3382/ps/pey439.

Azhar, M., Rahardja, D. P., \& Pakiding, W. (2016). Embryo development and post-hatch performances of kampung chicken by in ovo feeding of LArginine. Media Peternakan, 39(3), 168-172. 10.5398/medpet.2016.39.3.168.

Beck, C. N., McDaniel, C. D., Wamsley, K. G. S., \& Kiess, A. S. (2019). The potential for inoculating Lactobacillus animalis and Enterococcus faecium alone or in combination using commercial in ovo technology without negatively impacting hatch and post-hatch performance. Poultry Science, 98 (12), $7050-7062$. $10.3382 / \mathrm{ps} / \mathrm{pez} 441$.

Berrocoso, J. D., Kida, R., Singh, A. K., Kim, Y. S., \& Jha, R. (2016). Effect of in ovo injection of raffinose on growth performance and gut health parameters of broiler chicken. Poultry Science, pew430. 10.3382/ps/pew430.

Bhanja, S. K., Goel, A., Pandey, N., Mehra, M., Majumdar, S., \& Mandal, A. B. (2014). In ovo carbohydrate supplementation modulates growth and immunity-related genes in broiler chickens. Journal of Animal Physiology and Animal Nutrition, 99(1), 163-173. 10.1111/jpn.12193.

Biria, A., Navidshad, B., Mirzaei Aghjehgheshlag, F., Nikbin, S. (2020). Effect of in ovo Supplementation of Nano Zinc Oxide Particles on Hatchability and Post-Hatch Immune System of Broiler Chicken. Iranian Journal of Applied Animal Science, 10(3), 547-553. http://ijas.iaurasht.ac.ir/article_675497.html.

Bogucka, J., Dankowiakowska, A., Elminowska-Wenda, G., Sobolewska, A., Szczerba, A., \& Bednarczyk, M. (2016). Effects of prebiotics and synbiotics delivered in ovo on broiler small intestine histomorphology during the first days after hatching. Folia Biologica,64(3), 131-143. 10.3409/fb64_3.131.

Castañeda, C. D., Dittoe, D. K., Wamsley, K. G. S., McDaniel, C. D., Blanch, A., Sandvang, D., \& Kiess, A. S. (2020). In ovo inoculation of an Enterococcus faecium - based product to enhance broiler hatchability, live performance, and intestinal morphology. Poultry Science, 99(11), 61636172. 10.1016/j.psj.2020.08.002. 
Coskun, I., Akkan, A., \& Erener, G. (2018). Effects of in ovo injection of lysine and methionine into fertile broiler (parent stock) eggs on hatchability, growth performance, caecum microbiota, and ileum histomorphology. Revista Brasileira de Zootecnia, 47. 10.1590/rbz4720170220.

Costa, F. G., Silva, J. H. V., Goulart, C. C., Nogueira, E. T. \& Sá, L. M. (2014). Exigência de aminoácidos para aves. In: Nutrição de não ruminantes. Jaboticabal: Funep-Unesp, cap. 4, 240-261.

Dal Pont, G. C., Goes, E. C., Araujo, R. A., Oliveira, S. G., Rocha, C., \& Maiorka, A. (2019). Glycerol inoculation in eggs of young broiler breeders at different embryonic periods. Poultry Science, 98(9), 3989-3993. 10.3382/ps/pez141.

Dal' Alba, G. M. (2018). Efeito da nutrição in ovo de mel de abelhas apis mellifera sobre o desempenho e rendimento de carcaça de frangos de corte. Dissertação (Mestrado) - Universidade do Estado de Santa Catarina, Chapecó. p. 49. http://sistemabu.udesc.br/pergamumweb/vinculos/000047/0000477f.pdf.

Dal'Alba, G. M., Melek, C., Schneider, M., Deolindo, G. L., Boiago, M. M., Faria, G. A., Stefani, L. M., Silva, A. S. da., \& Araujo, D. N. (2020). Nutrição in ovo usando mel: efeitos sobre eclodibilidade, desempenho e rendimento de carcaça em frangos de corte. Research, Society and Development, [S. 1.], 9(8), e43985178. 10.33448/rsd-v9i8.5178.

Damasceno, J. L., Cruz, F. G. G., Melo, R. D., Feijó, J. C., Rufino, J. P. F., Valentins, F. M., \& Oliveira, J. P. C. (2017). Inoculação de proteína isolada de soja em ovos embrionados oriundos de matrizes semipesadas com diferentes idades. Arquivo Brasileiro de Medicina Veterinária e Zootecnia, 69(5),1259-1266. $10.1590 / 1678-4162-9069$

Dankowiakowska, A., Bogucka, J., Sobolewska, A., Tavaniello, S., Maiorano, G., \& Bednarczyk, M (2019). Effects of in ovo injection of prebiotics and synbiotics on the productive performance and microstructural features of the superficial pectoral muscle in broiler chickens. Poultry Science, 98(10), 51575165. $10.3382 / \mathrm{ps} / \mathrm{pez} 202$.

De Meyer, F., Eeckhaut, V., Ducatelle, R., Dhaenens, M., Daled, S., Dedeurwaerder, A., De Gussem, M., Haesebrouck, F., Deforce, D., \& Van Immerseel, F. (2019). Host intestinal biomarker identification in a gut leakage model in broilers. Veterinary Research, 50(1), 14. 10.1186/s13567-019-0663-x.

Dorigam, J. C. P. (2016). Relação ideal dos aminoácidos essenciais para mantença, crescimento e produção de aves. Tese (Doutorado em Zootecnia) Universidade Estadual Paulista, Faculdade de Ciências Agrárias e Veterinárias, Jaboticabal. p. 193. https://repositorio.unesp.br/handle/11449/137840.

Ebrahimi, M., Janmohammadi, H., Daghigh Kia, H., Moghaddam, G., Rajabi, Z., Abbas Rafat, S., \& Javanmard, A. (2017). The effect of L-lysine in ovo feeding on body weight characteristics and small intestine morphology in a day-old Ross broiler chicks. Revue de Medecine Veterinaire, 168, 116-125. https://www.revmedvet.com/2017/RMV168_116_125.pdf.

El-Deep, M. H., Amber, K. A., Elgendy, S., Dawood, M. A. O., \& Zidan, A. (2020). In ovo injection of nano-selenium spheres mitigates the hatchability, histopathology image and immune response of hatched chicks. Journal of Animal Physiology and Animal Nutrition, 104(5), 1392-1400. 10.1111/jpn.13379

El-Moneim, A. E.-M. E. A., El-Wardany, I., Abu-Taleb, A. M., Wakwak, M. M., Ebeid, T. A., \& Saleh, A. A. (2019). Assessment of In Ovo Administration of Bifidobacterium bifidum and Bifidobacterium longum on Performance, Ileal Histomorphometry, Blood Hematological, and Biochemical Parameters of Broilers. Probiotics and Antimicrobial Proteins, 12, 439-450. 10.1007/s12602-019-09549-2.

El-Senousey, H. K., Chen, B., Wang, J. Y., Atta, A. M., Mohamed, F. R., \& Nie, Q. H. (2017). In ovo injection of ascorbic acid modulates antioxidant defense system and immune gene expression in newly hatched local Chinese yellow broiler chicks. Poultry Science, 97(2), 425-429. 10.3382/ps/pex310.

Gao, T., Zhao, M. M., Li, Y. J., Zhang, L., Li, J. L., Yu, L. L., Gao, F., \& Zhou, G. H. (2017). Effects of in ovo feeding of L-arginine on the development of digestive organs, intestinal function and post-hatch performance of broiler embryos and hatchlings. Journal of Animal Physiology and Animal Nutrition, 102(1), 166-175. 10.1111/jpn.12724.

Garcia, D. A., \& Gomes, D. E. (2019). A Avicultura Brasileira e Os Avanços Nutricionais. Revista Científica, 1(1). http://revistas.unilago.edu.br/index.php/revista-cientifica/article/view/167.

Givisiez, P. E. N., Moreira Filho, A. L. B., Santos, M. R. B., Oliveira, H. B., Ferket, P. R., Oliveira, C. J. B., \& Malheiros, R. D. (2020). Chicken embryo development: metabolic and morphological basis for in ovo feeding technology. Poultry Science, 99(12), 6774-6782. 10.1016\%2Fj.psj.2020.09.074.

Gonçalves, F. M., Santos, V. L., Contreira, C. L., Farina, G., Kreuz, B. S., Gentilini, F. P., Anciuti, M. A., \& Rutz, F. (2013). Nutrição in ovo: estratégia para nutrição de precisão em sistemas de produção avícola. Archivos de Zootecnia, 62(237), 54-55. 10.21071/az.v62i237.1956.

Gonzalez, J. M., \& Jackson, A. R. (2020). In ovo feeding of nicotinamide riboside affects broiler pectoralis major muscle development 1,2. Translational Animal Science, 4(3). 10.1093/tas/txaa126.

Han, G., Ouchi, Y., Hirota, T., Haraguchi, S., Miyazaki, T., Arakawa, T., Masuhara, N., Mizunoya, W., Tatsumi, R., Tashiro, K., Bungo, T., Furuse, M., \& Chowdhury, V. S. (2020). Effects of L-leucine in ovo feeding on thermotolerance, growth and amino acid metabolism under heat stress in broilers. Animal, 14(8), 1701-1709. 10.1017/S1751731120000464.

Hollemans, M. S., Vries, S., Lammers, A., \& Clouard, C. (2018). Effects of early nutrition and transport of 1-day-old chickens on production performance and fear response. Poultry science, 97(7), 2534-2542. 10.3382/ps/pey106.

Jha, R., Singh, A. K., Yadav, S., Berrocoso, J. F. D., \& Mishra, B. (2019). Early nutrition programming (in ovo and post-hatch feeding) as a strategy to modulate gut health of poultry. Frontiers in Veterinary Science, 6, 82. 10.3389/fvets.2019.00082.

Joshua, P. P., Valli, C., \& Balakrishnan, V. (2016). Effect of in ovo supplementation of nano forms of zinc, copper, and selenium on post-hatch performance of broiler chicken. Veterinary world, 9(3), 287. 10.14202\%2Fvetworld.2016.287-294.

Kadam, M. M., Barekatain, M. R., Bhanja, S. K., \& Iji, P. A. (2013). Prospects of in ovo feeding and nutrient supplementation for poultry: The science and commercial applications-a review. Journal of the Science of Food and Agriculture, 93, 3654-3661. 10.1002/jsfa.6301. 
Kanagaraju, P. \& Rathnapraba, S. (2019). Effect of in-ovo injection of glucose and egg white protein on the production performance and gut histomorphometry of broiler chicken. Indian Journal of Animal Research, 53(5), 675-679. 10.18805/ijar.B-3555.

Kermanshahi, H., Golian, A., Khodambashi Emami, N., Daneshmand, A., Ghofrani Tabari, D., \& Ibrahim, S. A. (2016). Effects of in ovo injection of threonine on hatchability, intestinal morphology, and somatic attributes in Japanese quail (Coturnix japonica). Journal of Applied Animal Research, 45(1), 437-441. 10.1080/09712119.2016.1206902.

Leitão, R. A., Leandro, N. S. M., Stringhini, J. H., Café, M. B., Matos, M. S., \& Andrade, M. A. (2014). Inoculação de maltose e/ou sacarose em ovos leves embrionados. Ciência Animal Brasileira, Goiânia, 15(1), 55-63. 10.5216/cab.v15i1.13974.

Li, T., Castañeda, C. D., Miotto, J., McDaniel, C., Kiess, A. S., \& Zhang, L. (2020). Effects of in ovo probiotic administration on the incidence of avian pathogenic Escherichia coli in broilers and na Evaluation on their virulence and antimicrobial resistance properties. Poultry Science. doi10.3382/ps/pez202.

Lima, M. D., Lopes, I. M. G., Silva, K. F., Miranda, H. A. F., Almeida, A. C., \& Duarte, E. R. (2020). Uso de aditivos em dietas para leitões em fase de creche: uma revisão. Research, Society and Development, [S. 1.], 9(12), e26491211081. 10.33448/rsd-v9i12.11081.

Madej, J. P., \& Bednarczyk, M. (2015). Effect ofin ovo-delivered prebiotics and synbiotics on the morphology and specific immune cell composition in the gut-associated lymphoid tissue. Poultry Science, 95(1), 19-29. 10.3382/ps/pev291.

Maia, G. D. (2018). Avaliação da inoculação de aminoácidos in ovo sobre a taxa de eclosão e qualidade de pintos de corte. Trabalho de Conclusão de Curso (Bacharelado em Zootecnia) - Universidade Tecnológica Federal do Paraná. Dois Vizinhos. p. 35. http://repositorio.utfpr.edu.br/jspui/handle/1/11328.

Martins, B. B. Probiótico administrado em embriões e pintos de frangos de corte na redução da colonização por Salmonella Heideberg e integridade entérica. Tese de Doutorado. Programa de Pós Graduação em Zootecnia, Universidade Estadual Paulista - Faculdade de Medicina Veterinária e Zootecnia, Câmpus de Botucatu, SP. p. 118. https://repositorio.unesp.br/handle/11449/158308.

Martins, R. A., Almeida A., \& Andrey, S. (2018). Importância dos aminoácidos na nutrição de frangos de corte. Revisão de Literatura. Revista Brasileira de Higiene e Sanidade Animal, 12(4), 539-554. http://www.higieneanimal.ufc.br/seer/index.php/higieneanimal/article/view/484

Miśta, D., Króliczewska, B., Pecka-Kiełb, E., Kapuśniak, V., Zawadzki, W., Graczyk, S., Kowalczyk, A., Lukaszewicz, E., \& Bednarczyk, M. (2017). Effect of in ovo injected prebiotics and synbiotics on the caecal fermentation and intestinal morphology of broiler chickens. Animal Production Science, 57(9), 18841892. 10.1071/AN16257.

Moreira Filho, A. L. B., Oliveira, C. J. B. de., Freitas Neto, O. C. de., Leon, C. M. C. G. de., Saraiva, M. M. S., Andrade, M. F. S., White, B., \& Givisiez, P. E. N. (2018). Intra-Amnionic Threonine Administered to Chicken Embryos Reduces Salmonella Enteritidis Cecal Counts and Improves Post hatch Intestinal Development. Journal of Immunology Research, 1-10. 10.1155/2018/9795829.

Neveling, D. P., \& Dicks, L. M. T. (2020). Probiotics: an Antibiotic Replacement Strategy for Healthy Broilers and Productive Rearing. Probiotics and Antimicrobial Proteins. 10.1007/s12602-020-09640-z.

Neves, D. G. (2019). In ovo injection of glycerol and insulin-like growth factor (IGF-I) for broilers. Tese (Doutorado em Zootecnia) - Universidade Federal de Lavras, Lavras. p. 111. https://pesquisa.bvsalud.org/portal/resource/pt/vtt-212692.

Neves, D. G. das., Retes, P. L., Alves, V. V., Pereira, R. S. G., Bueno, Y. do C., Alvarenga, R. R., \& Zangeronimo, M. G. (2020). In ovo injection with glycerol and insulin-like growth factor (IGF-I): hatchability, intestinal morphometry, performance, and carcass characteristics of broilers. Archives of Animal Nutrition, 74(4), 325-342. 10.1080/1745039X.2020.17473.

Neves, D. G., Retes, P. L., Rocha, R. R., Ferreira, L. G., Naves, L. P., Alvarenga, R. R., Fassani, E. J., Pereira, L. J., Sousa, R. V., \& Zangeronimo, M. G. (2016). Effects of in ovo feeding with glycerol for broilers. Journal of Animal Physiology and Animal Nutrition, 101(3), 434-440. 10.1111/jpn.12578.

Nunes, J. R. da. S., Cruz, F. G. G., Chagas, E. O., Maquiné, L. C., Melo, J. B. S., \& Miller, W. P. M. (2018). Inoculação de glicose e clara de ovo em ovos embrionados- Relato de caso. Revista Científica de Avicultura e Suinocultura, 4(1). https://periodicos.ufam.edu.br/index.php/RECAS/article/view/4949.

Oladokun, S., Koehler, A., MacIsaac, J., Ibeagha-Awemu, E. M., Adewole, D. I. (2020). Bacillus subtilis delivery route: effect on growth performance, intestinal morphology, cecal short-chain fatty acid concentration and cecal microbiota in broiler chickens. Poultry Science. S0032579120308269. 10.1016/j.psj.2020.10.063.

Oliveira, T. F. B., Bertechini, A. G., Bricka, R. M., Kim, E. J., Gerard, P. D., \& Peebles, E. D. (2015). Effects of in ovo injection of organic zinc, manganese, and copper on the hatchability and bone parameters of broiler hatchlings. Poultry Science, 94(10), 2488-2494. 10.3382/ps/pev248.

Omidi, S., Ebrahimi, M., Janmohammadi, H., Moghaddam, G., Rajabi, Z., \& Hosseintabar-Ghasemabad, B. (2019). The impact of in ovo injection of 1 -arginine on hatchability, immune system and caecum microflora of broiler chickens. Journal of Animal Physiology and Animal Nutrition, 104(1), 178-185. $10.1111 /$ jpn.13222.

Peebles, E. D. (2018). In ovo applications in poultry: a review. Poultry science, 97(7), 2322-2338. 10.3382/ps/pey081.

Pereira, A. S., Shitsuka, D. M., Parreira, F. J., \& Shitsuka, R. (2018). Metodologia da pesquisa científica. UFSM. http://repositorio.ufsm.br/handle/1/15824.

Pruszynska-Oszmalek, E., Kolodziejski, P. A., Stadnicka, K., Sassek, M., Chalupka, D., Kuston, B., Nogowski, L., Mackowiak, P., Maiorano, G., Jankowski, J., \& Bednarczyk, M. (2015). In ovo injection of prebiotics and synbiotics affects the digestive potency of the pancreas in growing chickens. Poultry Science, 94(8), 1909-1916. 10.3382/ps/pev162.

Reis, T. L. (2018). Nutrição precoce de pintos de corte. Ciência Animal, 28(1), 82-97. http://www.uece.br/cienciaanimal/dmdocuments/v28p82-97.pdf.

Reis, T. L., \& Vieites, F. M. (2019). Antibiótico, prebiótico, probiótico e simbiótico em rações de frangos de corte e galinhas poedeiras. Ciência Animal, 29(3), 133-147. https://pesquisa.bvsalud.org/portal/resource/pt/vti-745192. 
Rocha, J. S. R. (2011). Efeito da cantaxantina dietética para matrizes pesadas com idade avançada e do período de armazenamento dos ovos sobre a fertilidade, rendimento de incubação, nutrientes da gema e desenvolvimento embrionário. Tese (Doutorado em Zootecnia) - Universidade Federal de Minas Gerais, Escola de Veterinária. p. 80. http://hdl.handle.net/1843/BUOS-8FWL4A.

Rosalen, K., Camerini, N. L., Piazzetta, H. V. L., Berenchtein, B., \& Mota, D. A. (2020). Avaliação da temperatura corporal de frangos de corte usando imagens termográficas. Brazilian Journal of Development, 6(6), 42176-42184. 10.34117/bjdv6n6-671.

Roto, S. M., Kwon, Y. M., \& Ricke, S. C. (2016). Applications of In Ovo Technique for the Optimal Development of the Gastrointestinal Tract and the Potential Influence on the Establishment of Its Microbiome in Poultry. Frontiers in Veterinary Science, 3. 10.3389/fvets.2016.00063

Saeed, M., Babazadeh, D., Naveed, M., Alagawany, M., Abd El-Hack, M. E., Arain, M. A., Tiwari, R., Sachan, S., Karthik, K., Dhama, K., Elnesr, S. S., \& Chao, S. (2019). In ovo delivery of various biological supplements, vaccines and drugs in poultry: Current knowledge. Journal of the Science of Food and Agriculture, 99(8), 3727-3739. 10.1002/jsfa.9593.

Sahr, W. B. K., Odutayo, O. J., Sogunle, O. M., Ayo-Ajasa, O. Y., Fafiolu, A. O., \& Fatunmbi, F. A. (2020). Effects of in ovo injection og inorganic salts of $\mathrm{Zn}, \mathrm{Cu}$, and $\mathrm{Mn}$ on hatching traits and post-hatch performance of broiler chickens in the tropics. Nigerian Journal of Animal Science, 22(1), 113-125. https://www.ajol.info/index.php/tjas/article/view/197156.

Schmidt, M., Gomes, P. C., Rostagno, H. S., Albino, L. F. T., Nunes, R. V., \& Mello, H. H. C. M. (2011). Níveis nutricionais de metionina + cistina digestível para poedeiras leves no segundo ciclo de produção. Revista Brasileira de Zootecnia, 40(1), 142-147. 10.1590/S1516-35982011000100020.

Sharma, J. M., \& Witter, R. L. (1983). Embryo vaccination against Marek's disease with serotypes 1, 2 and 3 vaccines administered singly or in combination. Avian diseases, 27(2), 453-463. 10.2307/1590171.

Shokraneh, M., Sadeghi, A. A., Mousavi, S. N., Esmaeilkhanian, S., \& Chamani, M. (2020). Effects of in ovo injection of nano-selenium and nano-zinc oxide and high eggshell temperature during late incubation on antioxidant activity, thyroid and glucocorticoid hormones and some blood metabolites in broiler hatchlings. Acta Scientiarum Animal Sciences, 42(1), e46029. 10.4025/actascianimsci.v42i1.46029.

Silva, I. G. D. da. (2016). Efeito da inoculação in ovo de probiótico e produto de exclusão competitiva em frangos de corte desafiados com Salmonella Heidelberg. Dissertação (Mestrado) - UNESP- Botucatu. p. 58. https://repositorio.unesp.br/handle/11449/143436.

Siwek, M., Slawinska, A., Stadnicka, K., Bogucka, J., Dunislawska, A., \& Bednarczyk, M. (2018). Prebiotics and synbiotics - in ovo delivery for improved lifespan condition in chicken. BMC Veterinary Research, 14(1). 10.1186/s12917-018-1738-z.

Skjøt-Rasmussen, L., Sandvang, D., Blanch, A., Nielsen, J. M., Styrishave, T., Schnabl, J., Brockmann, E., Beck, C. N., \& Kiess, A. S. (2019). Post hatch recovery of a probiotic Enterococcus faecium strain in the yolk sac and intestinal tract of broiler chickens after in ovo injection. FEMS Microbiology Letters, 366(7), 13. 10.1093/femsle/fnz078.

Slawinska, A., Plowiec, A., Siwek, M., Jaroszewski, M., \& Bednarczyk, M. (2016). Long-Term Transcriptomic Effects of Prebiotics and Synbiotics Delivered In Ovo in Broiler Chickens. PLOS ONE, 11(12), e0168899. 10.1371/journal.pone.0168899.

Slawinska, A., Zampiga, M., Sirri, F., Meluzzi, A., Bertocchi, M., Tavaniello, S., \& Maiorano, G. (2019). Impact of galactooligosaccharides delivered in ovo on mitigating negative effects of heat stress on performance and welfare of broilers. Poultry Science, 99(1), 407-415. 10.3382/ps/pez512.

Sobolewska, A., Elminowska-Wenda, G., Bogucka, J., Dankowiakowska, A., Kułakowska, A., Szczerba, A., Stadnicka, K., Szpinda, M., \& Bednarczyk, M. (2017). The influence of in ovo injection with the prebiotic DiNovo ${ }^{\circledR}$ on the development of histomorphological parameters of the duodenum, body mass and productivity in large-scale poultry production conditions. Journal of Animal Science and Biotechnology, 8(1), 45. 10.1186/s40104-017-0176-2.

Sun, X., Lu, L., Liao, X., Zhang, L., Lin, X., Luo, X., \& Ma, Q. (2018). Effect of In Ovo Zinc Injection on the Embryonic Development and EpigeneticsRelated Indices of Zinc-Deprived Broiler Breeder Eggs. Biological Trace Element Research, 185(2), 456-464. 10.1007/s12011-018-1260-y.

Tainika, B., \& Şekeroğlu, A. (2020). In ovo Feeding Technology for Optimization of Incubation and Hatching in Broiler Chickens. Turkish Journal of Agriculture - Food Science and Tecnology, 8. 10.24925/turjaf.v8isp1.183-186.4086.

Tavaniello, S., Slawinska, A., Prioriello, D., Petrecca, V., Bertocchi, M., Zampiga, M., Salvatori, M., \& Maiorano, G. (2019). Effect of galactooligosaccharides delivered in ovo on meat quality traits of broiler chickens exposed to heat stress. Poultry Science. 99(1), 612-619. 10.3382/ps/pez556.

Teymouri, B., Ghiasi Ghalehkandi, J., Hassanpour, S., \& Aghdam-Shahryar, H. (2019). Effect of In Ovo Feeding of the Vitamin B12 on Hatchability, Performance and Blood Constitutes in Broiler Chicken. International Journal of Peptide Research and Therapeutics, 26, 381-387. 10.1007/s10989-01909844-0.

Toghyani, M., Tahmasebi, S., Modaresi, M., \& Ale Saheb Fosoul, S. S. (2018). Effect of arginine and threonine in ovo supplementation on immune responses and some serum biochemical attributes in broiler chickens. Italian Journal of Animal Science, 18(1), 342-349. 10.1080/1828051x.2018.1529545.

Torres, C. A., \& Korver, D. R. (2018). Influences of trace mineral nutrition and maternal flock age on broiler embryo bone development. Poultry science, 97(8), 2996-3003. 10.3382/ps/pey136.

Van Milgen, J., \& Dourmad, J.-Y. (2015). Concept and application of ideal protein for pigs. Journal of Animal Science and Biotechnology, 6(1). 10.1186/s40104-015-0016-1.

Wang, H., Ni, X., Qing, X., Liu, L., Lai, J., Khalique, A., Guangyao, L., Kangcheng, P., Bo, J., \& Zeng, D. (2017). Probiotic Enhanced Intestinal Immunity in Broilers against Subclinical Necrotic Enteritis. Frontiers in Immunology, 8. 10.3389/fimmu.2017.01592.

Wang, J., Lin, J., Wang, J., Wu, S., Qi, G., Zhang, H., \& Song, Z. (2020). Effects of in ovo feeding of N-acetyl-L-glutamate on early intestinal development and growth performance in broiler chicken. Poultry Science, 99(7), 3583-3593. 10.1016/j.psj.2020.04.003.

Wijnen, H. J., Molenaar, R., van Roovert-Reijrink, I. A. M., van der Pol, C. W., Kemp, B., \& van den Brand, H. (2020). Effects of incubation temperature pattern on broiler performance. Poultry Science, 99(8), 3897-3907. 10.1016/j.psj.2020.05.010. 
Research, Society and Development, v. 10, n. 2, e38810212307, 2021

(CC BY 4.0) | ISSN 2525-3409 | DOI: http://dx.doi.org/10.33448/rsd-v10i2.12307

Yair, R., Shahar, R., \& Uni, Z. (2015). In ovo feeding with minerals and vitamin D3 improves bone properties in hatchlings and mature broilers. Poultry Science, 94(11), 2695-2707. 10.3382/ps/pev252.

Yu, L. L., Gao, T., Zhao, M. M., Lv, P. A., Zhang, L., Li, J. L., Jiang, Y., Gao, F. \& Zhou, G. H. (2017). In ovo feeding of L-arginine alters energy metabolism in post-hatch broilers. Poultry Science, 97(1), 140-148. 10.3382/ps/pex272.

Zhang, H., Elliott, K. E. C., Durojaye, O. A., Fatemi, S. A., Schilling, M. W., \& Peebles, E. D. (2019). Effects of in ovo injection of L-ascorbic acid on growth performance, carcass composition, plasma antioxidant capacity, and meat quality in broiler chickens1,2,3. Poultry Science, 98(9), 3617-3625. $10.3382 / \mathrm{ps} / \mathrm{pez} 173$.

Zhu, Y. F., Li, S. Z., Sun, Q. Z., \& Yang, X. J. (2019). Effect of in ovo feeding of vitamin C on antioxidation and immune function of broiler chickens. Animal, 1-7. 10.1017/s1751731118003531.

Zhu, Y., Li, S., Duan, Y., Ren, Z., Yang, X., \& Yang, X. (2020). Effects of in ovo feeding of vitamin C on post-hatch performance, immune status and DNA methylation-related genes expression in broiler chickens. British Journal of Nutrition, 124(9), 903-911. 10.1017/s000711452000210x. 